\title{
Absence of Edge Localized Moments in the Doped Spin-Peierls System $\mathrm{CuGe}_{1-x} \mathrm{Si}_{x} \mathrm{O}_{3}$
}

\author{
J. Kikuchi, ${ }^{1}$ 用 T. Matsuoka, ${ }^{1}$ K. Motoya,${ }^{1}$ T. Yamauchi, ${ }^{2}$ and Y. Ueda ${ }^{2}$ \\ ${ }^{1}$ Department of Physics, Science University of Tokyo, Yamazaki, Noda, Chiba 278-8510, Japan \\ ${ }^{2}$ Institute for Solid State Physics, University of Tokyo, Kashiwanoha, Kashiwa, Chiba 27r-8581, Japan
}

(Dated: August 2 2001)

\begin{abstract}
We report the observation of nuclear quadrupole resonance (NQR) of $\mathrm{Cu}$ from the sites near the doping center in the spin-Peierls system $\mathrm{CuGe}_{1-x} \mathrm{Si}_{x} \mathrm{O}_{3}$. The signal appears as the satellites in the $\mathrm{Cu}$ NQR spectrum, and has a suppressed nuclear spin-lattice relaxation rate indicative of a singlet correlation rather than an enhanced magnetic correlation near the doping center. Signal loss of $\mathrm{Cu}$ nuclei with no neighboring $\mathrm{Si}$ is also observed. We conclude from these observations that the doping-induced moments are not in the vicinity of the doping center but rather away from it.
\end{abstract}

PACS numbers: 76.60.-k, 75.10.Jm, 75.50.Ee, 75.40.Gb

Impurity-induced magnetism in low-dimensional quantum spin systems has been the subject of recent intense theoretical and experimental studies. The discovery of the first inorganic spin-Peierls (SP) material $\mathrm{CuGeO}_{3}$ [1] has opened up the field of experimental research into the impurity effects on the SP transition which is inherent in the spin- $\frac{1}{2}$ Heisenberg antiferromagnetic (AF) spin chains coupled with phonons. In-chain substitution or doping of $\mathrm{Zn}, \mathrm{Mg}, \mathrm{Ni}$ for $\mathrm{Cu}$ [2, 3, 4, 5] as well as off-chain substitution of Si for Ge [5, 6] have been studied intensively, and the temperature-concentration magnetic phase diagrams are now becoming established. Independently of the types of doping, a rapid decrease of the transition temperature $T_{\mathrm{SP}}$ followed by a disappearance of SP longrange order (LRO) is commonly observed. At the same time, there appears a new AF phase at low temperatures coexisting with SP-LRO at very small dopant concentrations. The coexistence of SP- and AF-LRO has attracted much attention because they had been thought to be exclusive.

The AF state induced by doping is rather unconventional. Although the AF order is commensurate, the size of an ordered moment is small and spatially varying [7. It is widely believed that the inhomogeneous AF-LRO results from the enhanced staggered correlation or the appearance of staggered moments near the doping center, of which existence is suggested from numerical calculations on a dimerized chain with open boundaries [8, 9, 10, phase-Hamiltonian approach assuming reduced dimerization near the impurity 11, and a soliton binding to the impurity due to interchain elastic couplings [12]. However, there is by now no experimental evidence that the staggered moments are really induced near the doping center in the SP system.

In this Letter, we present $\mathrm{Cu}$ NQR evidences that this is not the case in $\mathrm{CuGeO}_{3}$ doped with $\mathrm{Si}$. The enhanced moments appear not in the vicinity of Si atoms segmenting a linear chain but in between them, which comes from the flexibility of the lattice locating singlet bonds on the edges of an open segment.

Single crystals of $\mathrm{CuGe}_{1-x} \mathrm{Si}_{x} \mathrm{O}_{3}$ were grown by a float- ing zone method. The $\mathrm{Cu}$ NQR spectrum was taken under zero external field by integrating the spin-echo signal while changing the frequency point by point. Nuclear spin-lattice relaxation rate was measured by an inversion recovery method.

The $\mathrm{Cu}$ NQR spectra in the SP $(x=0,0.006$, $0.010,0.012)$ and uniform $(x=0.02,0.05)$ phases of $\mathrm{CuGe}_{1-x} \mathrm{Si}_{x} \mathrm{O}_{3}$ are shown in Fig. 11. As the Si content $x$ increases, satellite lines appear in addition to the original ${ }^{63,65} \mathrm{Cu}$ NQR lines similarly for both isotopes. From the isotopic ratios of resonance frequencies and intensities between the satellites, we associate the satellites with $\mathrm{Cu}$ sites having different crystallographic environment from that in the non-doped sample. Based on the intensity argument below, the satellites can be assigned as the signal from $\mathrm{Cu}$ nuclei with $\mathrm{Si}$ on the firstand/or second-neighbor Ge sites. Local change of lattice dimerization due to $\mathrm{Si}$ substitution is unlikely for the appearance of the satellites because the Cu NQR frequency in $\mathrm{CuGeO}_{3}$ is known to be insensitive to the lattice dimerization 13, 14.

We restrict the following discussion to the ${ }^{63} \mathrm{Cu}$ NQR. For convenience, we call the NQR line observed in the non-doped sample as the main (M) line. The satellite in the vicinity of the line $\mathrm{M}$ is called satellite 1 (S1) and the weaker one at lower frequencies the satellite 2 (S2). To compare the number of nuclei which contributes to each NQR line, we deconvoluted the spectrum by fitting to the sum of Gaussians. Figure 2(a) shows the $x$ dependence of the integrated intensities $I_{\mathrm{M}}, I_{\mathrm{S} 1}$ and $I_{\mathrm{S} 2}$ of the lines $\mathrm{M}$, $\mathrm{S} 1$ and $\mathrm{S} 2$ multiplied by temperature $T$ which are proportional to the number of nuclei contributing to the NQR line 15. The integrated intensities were extrapolated to $\tau=0$ ( $\tau$ being the radio-frequency pulse separation $)$ by measuring the spin-echo decay at the peak position of the lines M, S1 and S2, and were normalized by the number of ${ }^{63} \mathrm{Cu}$ nuclei included in each sample. We find a rapid decrease of $I_{\mathrm{M}} \cdot T$ and a moderate increase of $I_{\mathrm{S} 1} \cdot T$ and $I_{\mathrm{S} 2} \cdot T$ with $x$. Shown in Fig. 2(b) is the integrated intensity of all the NQR lines $I_{\text {total }}=I_{\mathrm{M}}+I_{\mathrm{S} 1}+I_{\mathrm{S} 2}$ times $T$ as a function of $x$. Surprisingly enough, $I_{\text {total }} \cdot T$ decreases 


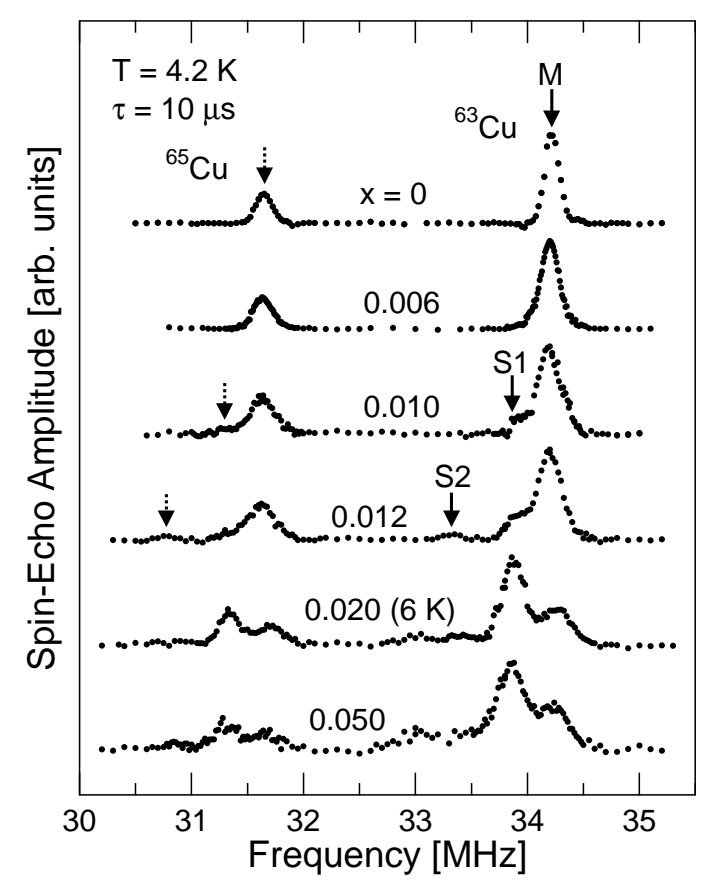

FIG. 1: $\mathrm{Cu}$ NQR spectrum in $\mathrm{CuGe}_{1-x} \mathrm{Si}_{x} \mathrm{O}_{3}$ at $4.2 \mathrm{~K}(x=$ 0.02 at $6 \mathrm{~K})$. The intensities are normalized by the signal maximum for each $x$. M, S1 and S2 with arrows (solid: ${ }^{63} \mathrm{Cu}$, dotted: ${ }^{65} \mathrm{Cu}$ ) denote the main line, satellite 1 and satellite 2 , respectively (see text).

with increasing $x$ in spite of the fact that we do not dilute the $\mathrm{Cu}$ site. This unambiguously demonstrates that a part of $\mathrm{Cu}$ nuclei is lost from NQR observation.

There seem to be two possible origins for the loss of NQR signal in $\mathrm{CuGe}_{1-x} \mathrm{Si}_{x} \mathrm{O}_{3}$. (i) Inhomogeneity of the electric-field gradient (EFG): Since an NQR frequency is highly sensitive to the local charge distribution, the signal may be wiped out if Si substitution introduces significant inhomogeneity of the EFG at the Cu site leading to a spread of NQR frequencies. It is expected that this is most effective, if any, near the doping center. (ii) Appearance of strongly-enhanced moments: Si substitution for Ge magnetically activates some of $\mathrm{Cu}^{2+}$ spins which otherwise is in a spin-singlet state. The signal cannot be observed if the nuclear spins on such $\mathrm{Cu}$ sites relax in a time shorter than the time domain of a pulsed NQR experiment $(>\mathrm{a}$ few $\mu \mathrm{s}$ ). It is difficult to predict the location of the induced moments in general, because it depends on the microscopic mechanisms for their appearance.

In order to know which part of the $\mathrm{Cu}$ nuclei becomes unobservable, we model the Si concentration dependence of the integrated intensity based on the probability argument. We assume that the NQR frequency is determined by the number of $\mathrm{Si}$ atoms on the first- and/or second-neighbor $\mathrm{Ge}$ sites for $\mathrm{Cu}$. The others are ne-

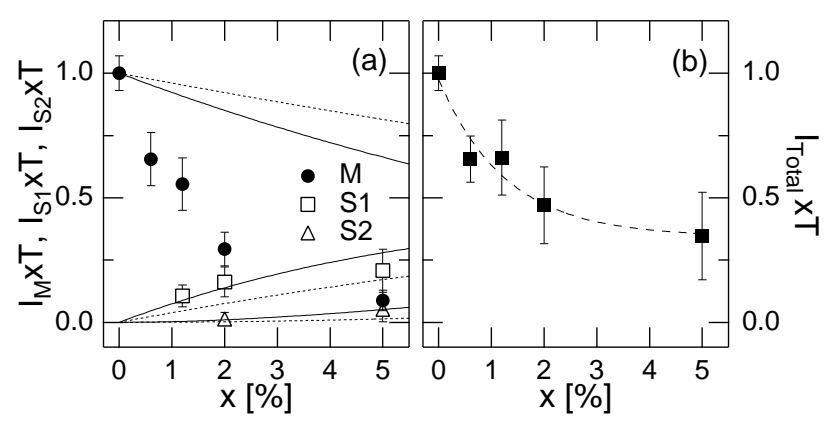

FIG. 2: Si concentration dependence of the integrated intensity of the ${ }^{63} \mathrm{Cu}$ NQR spectrum in $\mathrm{CuGe}_{1-x} \mathrm{Si}_{x} \mathrm{O}_{3}$ multiplied by temperature. The intensities are normalized by the number of ${ }^{63} \mathrm{Cu}$ nuclei in the $x=0$ sample. The intensity of each NQR line (a), and the total intensity (b). The dotted and solid lines in (a) are ${ }_{n} B_{k}(k=0,1,2)$ with $n=4$ and 8 , respectively. The dashed line in (b) is a guide to the eyes.

glected because they are relatively far apart. The satellite $1(2)$ hence corresponds to the $\mathrm{Cu}$ sites with one (two) Si atom(s) among four or eight neighboring Ge sites, and the main line to the $\mathrm{Cu}$ site without $\mathrm{Si}$ neighbors which is equivalent to the one in the non-doped sample. The probability of finding $\mathrm{Cu}$ atoms with $k(=0,1,2) \mathrm{Si}$ among $n$ (=4 or 8 ) neighboring Ge sites is nothing but the binomial probability ${ }_{n} B_{k}(x)={ }_{n} C_{k} x^{k}(1-x)^{n-k}$.

In Fig. 2(a) we compared ${ }_{4} B_{k}(x)$ and ${ }_{8} B_{k}(x)(k=0$, $1,2)$ with $I_{\mathrm{M}} \cdot T, I_{\mathrm{S} 1} \cdot T$ and $I_{\mathrm{S} 2} \cdot T$. It is apparent that the satellite intensities are best reproduced by taking $n=8$. On the other hand, $I_{\mathrm{M}} \cdot T$ decreases much faster than ${ }_{8} B_{0}(x)$. If we try to fit $I_{\mathrm{M}} \cdot T$, we must take $\sim 40 \mathrm{Ge}$ atoms up to the tenth-neighbor Ge sites. This, of course, cannot reproduce $I_{\mathrm{S} 1} \cdot T$ and $I_{\mathrm{S} 2} \cdot T$ and is quite unreasonable for the following reason. If the $\mathrm{Si}$ substitution effect so long-ranged, the satellite is considered to be severely broadened and may be wiped out because there are so many nonequivalent Si configurations corresponding to that satellite 16. In fact, we can observe the satellites with relatively narrow linewidths which contradicts with the above expectation. We therefore exclude the possibility that the rapid decrease of $I_{\mathrm{M}} \cdot T$ is due to long-range nature of the Si substitution effect on the EFG.

It is now clear that the signal loss occurs for the $\mathrm{Cu}$ sites without neighboring Si. This readily exclude the EFG inhomogeneity scenario for the loss of signal because there seems no disturbance to the EFG at such $\mathrm{Cu}$ sites. More importantly, local moments which appear in the vicinity of the impurity are clearly incompatible with the fact that the $\mathrm{Cu}$ sites near $\mathrm{Si}$ are observable as the satellites. A consistent explanation for the loss of signal from the main line would be the appearance of enhanced moments away from the doping center. Considering the effective interruption of linear chains by off-chain sub- 


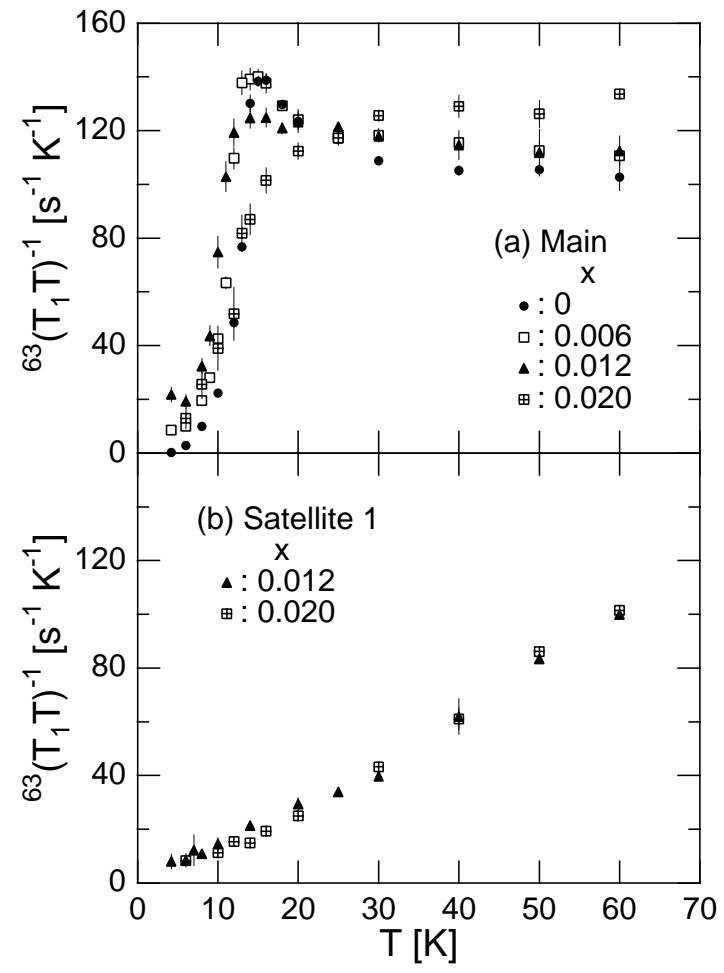

FIG. 3: Temperature dependence of the ${ }^{63} \mathrm{Cu}$ nuclear spinlattice relaxation rates ${ }^{63} 1 / T_{1} T$ for the line $\mathrm{M}(\mathrm{a})$, and the line $\mathrm{S} 1$ (b) in $\mathrm{CuGe}_{1-x} \mathrm{Si}_{x} \mathrm{O}_{3}$.

stitution [12], this implies, contrary to a common belief, that the local moments are induced in the middle of a segment rather than near the open edges.

The absence of impurity-induced moments near the doping center is also supported by the nuclear spin-lattice relaxation rate of $\mathrm{Cu}$. Non-exponential recovery of $\mathrm{Cu}$ nuclear magnetization $M(t)$ was observed at temperatures as high as $40 \mathrm{~K}$ for $x>0$ indicating spatiallynonuniform relaxation processes. We analyzed the recovery curves by fitting to the stretched exponential form $p(t) \equiv 1-M(t) / M(\infty) \propto \exp \left[-t / T_{1}-\left(t / \tau_{1}\right)^{1 / 2}\right]$ which works well in the system with dilute magnetic impurities [17]. In the present case, $1 / T_{1}$ can be interpreted as the relaxation due to the on-site electronic spin via the hyperfine coupling and $1 / \tau_{1}$ the relaxation resulting from the impurity-induced moments via the much weaker classical dipolar coupling. Figure 3 shows the $T$ dependence of ${ }^{63} \mathrm{Cu}$ nuclear spin-lattice relaxation rates ${ }^{63} 1 / T_{1} T(\mathrm{M})$ and ${ }^{63} 1 / T_{1} T(\mathrm{~S} 1)$ for the lines $\mathrm{M}$ and $\mathrm{S} 1[18$. It is evident from comparison of the absolute values of ${ }^{63} 1 / T_{1} T$ that $\mathrm{Cu}^{2+}$ spins near $\mathrm{Si}$ are magnetically less active than those without Si nearby.

We can trace the size effect on the dynamics of an open SP chain via $1 / T_{1}(\mathrm{M})$ and $1 / T_{1}(\mathrm{~S} 1)$ which probe bulkand edge-spin fluctuations, respectively. Let us look at $1 / T_{1}(\mathrm{M})$ first. In the uniform (U) phase of $\mathrm{CuGeO}_{3}$,

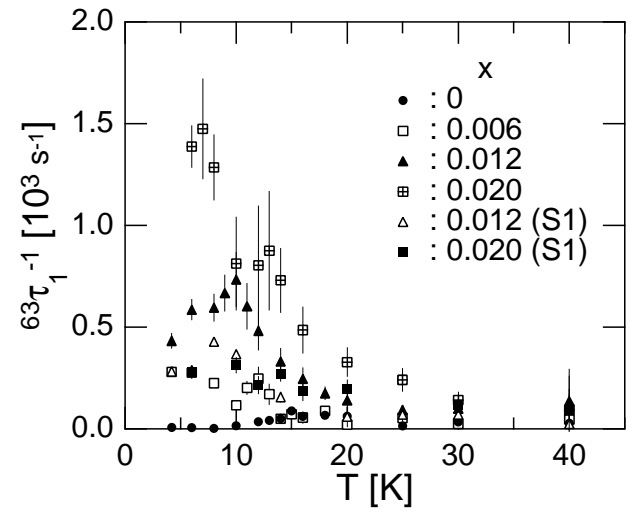

FIG. 4: Temperature dependence of the relaxation rate ${ }^{63} 1 / \tau_{1}$ due to impurity-induced moments in $\mathrm{CuGe}_{1-x} \mathrm{Si}_{x} \mathrm{O}_{3}$.

the $T$ dependence of $1 / T_{1} T$ at the $\mathrm{Cu}$ site is understood as being contributed by the nearly $T$-independent uniform (wave vector $q=0$ ) mode and the staggered $(q=\pi)$ mode showing $T^{-1}$-like divergence at low $T[14$. As shown in Fig. $3(\mathrm{a})$, the low- $T$ increase of ${ }^{63} 1 / T_{1} T(\mathrm{M})$ due to the staggered contribution is rounded off by doping and is completely suppressed in the $x=0.02$ sample in spite of the fact that the wave vector $q=\pi$ characterizes the low- $T$ AF-LRO. In addition, ${ }^{63} 1 / T_{1}(\mathrm{M})$ does not exhibit critical divergent behavior typical for threedimensional magnetic ordering even at low $T$. These observations suggest that the observed bulk spins do not play an active role for the appearance of AF-LRO. In the SP state $(x \leq 0.012),{ }^{63} 1 / T_{1} T(\mathrm{M})$ is increased by doping. This may result from the reduction of the SP gap and/or the appearance of spin-wave-like excitations below the SP gap 19. Such an $x$ dependence of the relaxation rate is qualitatively the same as that observed in Mg-doped $\mathrm{CuGeO}_{3} 20$.

As a clear contrast, ${ }^{63} 1 / T_{1} T(\mathrm{~S} 1)$ is essentially $x$ independent which indicates that the dynamics of the edge spin is insensitive to not only the size of a segment but also the existence of SP-LRO. The most striking feature of the result is the large suppression of ${ }^{63} 1 / T_{1} T(\mathrm{~S} 1)$ from the bulk ${ }^{63} 1 / T_{1} T(\mathrm{M})$ which begins gradually below about $60 \mathrm{~K}$. Pouget and his co-workers have revealed from the x-ray diffuse scattering studies that in-chain pretransitional lattice fluctuations or local dimer correlations exist as high as $40 \mathrm{~K}$ in both pure and Si-doped $\mathrm{CuGeO}_{3}$ 21, 22. The suppression of the edge-spin fluctuations probed by ${ }^{63} 1 / T_{1} T(\mathrm{~S} 1)$ may be associated with such preformation of singlet dimers on the edges.

Figure 1 shows the $T$ dependence of the relaxation rate ${ }^{63} 1 / \tau_{1}$ due to the impurity-induced moments. Minor differences of ${ }^{63} 1 / \tau_{1}$ between the lines $\mathrm{M}$ and S1 are probably due to long-range nature of the dipolar coupling. ${ }^{63} 1 / \tau_{1}$ is enhanced by doping and exhibits a Curie-Weisslike increase at low $T$ but above $T_{\mathrm{SP}}$. The low- $T$ increase 
of ${ }^{63} 1 / \tau_{1}$ indicates slowing down of the impurity-induced moments, because $1 / \tau_{1}$ is roughly proportional to the correlation time of the impurity-induced moments 17.

Now that the absence of doping-induced moments next to an impurity or an open chain end becomes obvious, theories predicting local moments near the chain end are not relevant to describe the magnetic properties of $\mathrm{Si}$ doped $\mathrm{CuGeO}_{3}$. Though gradual, there is a clear tendency of the edge spins to become nonmagnetic much faster than the bulk spins when lowering temperature in the U phase. This suggests that in the SP phase, strong bonds in which spins experience most of the time in a singlet state are formed on the open edges. The key ingredient will be a coupling with phonons which can adjust dynamically a dimerization pattern so as to minimize the total energy of the spin and the lattice systems. It has recently been shown numerically that the lattice relaxation stabilizes the dimerization pattern with the strong bonds on the edges of an open chain [23, 24], although such a possibility has been pointed out from intuitive considerations [9, 10, 12. The dimerization in $\mathrm{Si}$-doped $\mathrm{CuGeO}_{3}$ should therefore be such that an open chain is "terminated" by the strong bonds. A defect of bond alternation or a soliton will then be formed in the middle of a segment with an odd number of sites, accompanying modulation of lattice dimerization and staggered polarization of magnetic moments around it. This latter causes a dramatic loss of NQR signal from the $\mathrm{Cu}$ sites with no neighboring Si depending on the soliton size in comparison with the size of a segment. Similar discussion may be applied to the system with in-chain nonmagnetic dopants such as $\mathrm{Zn}$ and $\mathrm{Mg}$ because the interruption of a chain is more direct. It is worth noting that the signal loss may occur in the even-numbered segment because of the formation of a soliton-antisoliton pair at finite temperatures [9, 23. This may be an origin for the more-than-half reduction of the intensity of the line $\mathrm{M}$ at higher doping levels from the model calculation.

The present ${ }^{63} \mathrm{Cu}$ relaxation data show that the local singlet correlation persists in the sample with no SPLRO. This renders support for the soliton picture of the $\mathrm{SP}$ transition in pure and doped $\mathrm{CuGeO}_{3}$ that the SPLRO disappears when the interchain coherence of the lattice distortion is lost rather than the dimerization itself [12, 22]. The rapid decrease of $T_{\mathrm{SP}}$ due to doping may result from the weakness of the interchain elastic coupling leaving solitons away from the impurities in spite of the "irregular" dimerization pattern of that segment [12]. As for AF-LRO, nonmagnetic nature of the edge site, suppressed bulk AF correlations and the absence of critical divergence of $1 / T_{1}$ suggest that both the edge and bulk spins play a passive role for magnetic ordering. What becomes critical is the fluctuation of impurity-induced moments in the middle of a segment, which allows one to view the AF-LRO in Si-doped $\mathrm{CuGeO}_{3}$ as arising from the interchain magnetic coupling between the impurityinduced moments.

We thank for valuable discussion with Y. Itoh and C. Yasuda. T. Fujii and S. Ishiguro are also acknowledged for experimental help.

* E-mail: kikuchi@ph.noda.sut.ac.jp

[1] M. Hase, I. Terasaki, and K. Uchinokura, Phys. Rev. Lett. 70, 3651 (1993).

[2] S. Oseroff et al., Phys. Rev. Lett. 74, 1450 (1995).

[3] M. Hase et al., J. Phys. Soc. Jpn. 65, 1392 (1996).

[4] T. Masuda et al., Phys. Rev. Lett. 80, 4566 (1998).

[5] B. Grenier et al., Phys. Rev. B 58, 8202 (1998).

[6] J. P. Lenard et al., Europhys. Lett. 30, 475 (1995).

[7] K. M. Kojima et al., Phys. Rev. Lett. 79, 503 (1997).

[8] G. B. Martins et al., Phys. Rev. Lett. 78, 3563 (1997).

[9] M. Laukamp et al., Phys. Rev. B 57, 10755 (1998).

[10] S. Watanabe and H. Yokoyama, J. Phys. Soc. Jpn. 68, 2073 (1999).

[11] H. Fukuyama, T. Tanimoto, and M. Saito, J. Phys. Soc. Jpn. 65, 1182 (1996).

[12] D. Khomskii, W. Geertsma, and M. Mostovoy, Czech. J. Phys. 46, 3239 (1996).

[13] J. Kikuchi et al., J. Phys. Soc. Jpn. 63, 872 (1994).

[14] M. Itoh et al., Phys. Rev. B 52, 3410 (1995).

[15] A. Abragam, Principles of Nuclear Magnetism (Oxford University, Oxford, 1961).

[16] There are 10 and 55 nonequivalent configurations of $\mathrm{Si}$ for the lines S1 and S2, respectively, if we take account of up to the tenth-neighbor Ge sites, whereas we have only 2 (S1) and 4 (S2) configurations in the present model.

[17] M. R. McHenry, B. G. Silbernagel, and J. H. Wernick, Phys. Rev. B 5, 2958 (1972).

[18] $T_{1}$ is defined as the time constant characterizing the exponentially-decaying part of $p(t)$ measured by NQR as described in the text. In Refs. 13] and [14], on the other hand, $T_{1}$ is defined as $p(t) \propto \exp \left(-3 t / T_{1}\right)$ in which the factor 3 is incorrect for the nonaxial EFG in $\mathrm{CuGeO}_{3}$.

[19] K. Hirota et al., J. Phys. Soc. Jpn. 67, 645 (1998).

[20] Y. Itoh et al., cont-mat/0107353 (unpublished).

[21] J. P. Pouget et al., Phys. Rev. Lett. 72, 4037 (1994); J. P. Schoeffel et al., Phys. Phys. B 53, 14971 (1996).

[22] J. P. Pouget, Eur. Phys. J. B 20, 321 (2001).

[23] P. Hansen et al., Phys. Rev. B 59, 13557 (1999).

[24] H. Onishi and S. Miyashita, J. Phys. Soc. Jpn. 69, 2634 (2000). 